\title{
Disponibilidade familiar de alimentos na Zona da Mata e Semi-Árido do Nordeste do Brasil
}

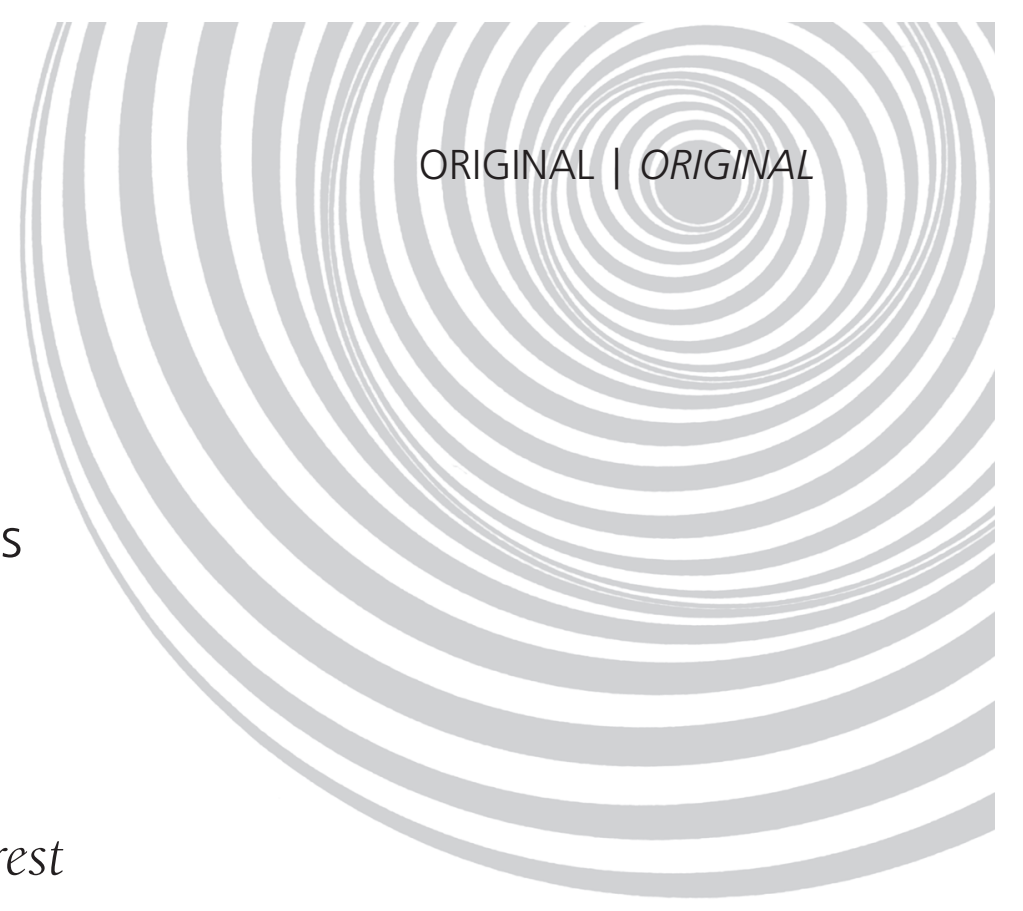

\author{
Household food availability in the Forest \\ Zone (Zona da Mata) and Semi-Arid \\ Brazilian Northeast regions
}

Mônica Maria OSÓRIO'

Marisilda de Almeida RIBEIRO'

Emilia Chagas COSTA'

Silvia Patrícia de Oliveira SILVA'

Carolina Estevam FERNANDES ${ }^{1}$

RE S U M O

\section{Objetivo}

Este trabalho teve como objetivo caracterizar a disponibilidade de alimentos em domicílios de Gameleira (PE) e São João do Tigre (PB), municípios das referidas regiões, considerando a freqüência dos alimentos, o número de pessoas no domicílio e a escolaridade do chefe da família.

\section{Métodos}

A amostra foi constituída de 502 e 458 famílias, em Gameleira (PE) e São João do Tigre (PB), respectivamente, de área urbana e rural de cada município. As informações, referentes aos sete dias anteriores à entrevista, foram fornecidas pelo responsável pela preparação dos alimentos.

\section{Resultados}

Verifica-se a disponibilidade de 33 alimentos em Gameleira (PE) e 30 em São João do Tigre (PB), em mais de $20 \%$ das famílias; restringindo-se a 16 e 15 alimentos nos dois municípios respectivamente, quando considerada a disponibilidade em $\mathbf{5 0 \%}$ das famílias. Os alimentos disponíveis na maioria dos domicílios possuíam alto valor energético e pertenciam aos grupos de cereais e leguminosas, em detrimento de hortaliças, frutas e produtos de origem animal. A disponibilidade alimentar sofreu pouca variação em decorrência do número de pessoas no domicílio e da escolaridade do chefe da família.

\footnotetext{
1 Universidade Federal de Pernambuco, Departamento de Nutrição, Programa de Pós-Graduação em Nutrição. Av. Moraes Rego, s/n., Cidade Universitária, 50670-901, Recife, PE, Brasil. Correspondência para/Correspondence to: M.M. OSÓRIO. E-mail:<mosorio@ufpe.br>.
} 
320 | M.M. OSÓRIO et al.

\section{Conclusão}

O estímulo ao aumento qualitativo e quantitativo da disponibilidade familiar de alimentos deve ser o foco dos esforços governamentais em programas sociais de combate à fome.

Termos de indexação: Alimentação. Consumo alimentar. Dieta. Fatores socioeconômicos. Inquéritos nutricionais.

\section{A B S T R A C T}

\section{Objective}

The purpose of this study was to determine household food availability in Gameleira (PE) and São João do Tigre (PB), municipalities in the abovementioned regions, taking into account the frequency of food consumption, number of people per household, and education level of the head of the household.

\section{Methods}

The sample consisted of 502 and 458 families in Gameleira (PE) and São João do Tigre (PB), respectively, living in both urban and rural areas. Information on food consumption during the seven days prior to the interview was provided by the person in charge of preparing the meals.

\section{Results}

The number of available food types was 34 in Gameleira and 30 in São João de Tigre for more than 20\% of the families in Gameleira (PE) and had 33 and 30 different food items per household, respectively; yet 50\% of the households in both municipalities had as few as 16 and 15 different food items per household, respectively. The food items available in most households were high-energy foods belonging to the grain and legume groups, instead of other vegetables, fruits and animal source foods. Basically, the number of people per household and the education level of the head of the household did not affect food availability.

\section{Conclusion}

Government efforts to fight hunger should focus on promoting an increase in the quality and quantity of food items per household.

Indexing terms: Feeding. Food consumption. Diet. Socioeconomic factors. Nutrition survey.

\section{N T R O D U ÇÃ O}

Verifica-se, ao longo da história, que um padrão dietético é determinado pelas características culturais, sociais, econômicas e políticas de uma sociedade, sendo os hábitos e as atitudes alimentares conseqüências destas. Mudanças nos padrões de dietas têm sido observadas como um fenômeno mundial, influenciado pelo atual processo de globalização, do qual o Brasil não se mostra ausente ${ }^{1-3}$.

Uma alimentação desequilibrada, quantitativa e/ou qualitativamente, por excesso ou escassez, tem sido comprovada cientificamente como sendo a principal causa de disfunções e doenças crônicas não transmissíveis, nas diversas regiões do mundo, inclusive em países em desenvolvimento. Tais enfermidades se mostram respon- sáveis por índices elevados de morbidade, incapacidade para o trabalho e mortalidade, com tendência crescente para as décadas vindouras ${ }^{4}$.

Ao reconhecer a insegurança alimentar como um fator de risco para a saúde do indivíduo, a Organização Mundial da Saúde orienta e recomenda estratégias a serem adaptadas à realidade de cada país, integrando ações intersetoriais, considerando como alimentação saudável, aquela capaz de debelar as carências nutricionais e prevenir doenças crônicas não transmissíveis, decorrentes de alimentação inadequada ${ }^{5,6}$.

Diante deste quadro, o Nordeste Brasileiro se apresenta como uma região internacionalmente conhecida e até estigmatizada, por sua geopolítica da desigualdade. Chamam a atenção as áreas do Semi-Árido, pela predominância de longos tempos de estiagem, e da Zona da Mata, 
dominada, desde o século XVI, pela monocultura da cana-de-açúcar, regiões que abrangem municípios de mais baixos Índices de Desenvolvimento Humano (IDH) do Brasil, consideradas, portanto, como um dos maiores desafios para o desenvolvimento da Política Nacional de Segurança Alimentar Nutricional7 ${ }^{7,8}$.

Diante das características multifacetárias que envolvem a alimentação e a nutrição humana, nelas implícita a prática alimentar, torna-se desejável e imprescindível a intensificação de estudos acerca dos alimentos disponíveis em âmbito familiar, em especial naqueles domicílios de menor poder aquisitivo ${ }^{6}$. No contexto destas populações, a identificação da disponibilidade familiar de alimentos torna-se instrumento fundamental para avaliação da insegurança alimentar e nutricional, demonstrando a sua magnitude, aliada às condições de pobreza da população exposta.

Frente ao exposto, o objetivo deste estudo é caracterizar a disponibilidade de alimentos em domicílios de municípios da Zona da Mata e do Semi-Árido do Nordeste, por área geográfica, considerando-se a freqüência dos alimentos segundo o número de pessoas no domicílio e a escolaridade do chefe da família. Este diagnóstico tem como premissa subsidiar a tomada de decisões de políticas públicas, além de disponibilizar uma base de referência para a avaliação da evolução do cenário alimentar destas áreas, diante de intervenções que venham a ser efetuadas pela atual Política Nacional de Segurança Alimentar e Nutricional.

\section{MÉ T O D O S}

Este trabalho foi parte da pesquisa Avaliação da Situação Alimentar e Nutricional e seus Fatores Determinantes em Conglomerados Urbanos e Rurais do Nordeste, a qual teve como objetivo avaliar a situação de saúde, alimentar e nutricional de populações radicadas nestes ecossistemas. Para tanto foram selecionados os municípios de Gameleira, na Zona da Mata Meridional do Estado de Pernambuco, e de São João do Tigre, no Semi-Árido da Paraíba, com Índices de Desenvolvimento Humano (IDH) de 0,590 e 0,527, respectivamente, considerados baixos, segundo informações constantes no Atlas de Desenvolvimento Humano no Brasil ${ }^{8}$. Trata-se de um estudo transversal, representando sítios sentinelas, para análise da situação basal e avaliação de resultados de eventuais intervenções na área.

A delimitação do sítio sentinela em cada município foi estabelecida pela composição de 250 famílias para cada uma das áreas geográficas (urbana e rural). O estudo, mediante visitas domiciliares, realizou levantamento de dados: ambientais, socioeconômicos, demográficos, de produção agrícola e agropecuária, alimentação, nutrição e saúde, além de políticas públicas direcionadas às famílias. No município de Gameleira foram entrevistadas 502 famílias, correspondendo a 251 domicílios em cada área geográfica. No município de São João do Tigre, foram entrevistadas 458 famílias, sendo 250 da área urbana e 208 da área rural, no período de 30 de março a 30 de junho de 2005.

O trabalho de campo deste estudo foi realizado por estudantes do Curso de Graduação em Nutrição da Universidade Federal de Pernambuco, previamente capacitados durante o treinamento, e que participaram do estudo piloto desenvolvido com a finalidade de verificar a viabilidade da aplicação do instrumento e a logística de campo.

Para identificação da disponibilidade de alimentos na família, objeto do trabalho ora apresentado, as informações foram fornecidas pela dona da casa ou por pessoa responsável pela preparação dos alimentos, em visitas domiciliares, nos períodos que antecediam as principais refeições. As informações foram registradas pelo entrevistador em um formulário contendo 122 alimentos distribuídos entre os seguintes grupos: cereais e derivados (14); leguminosas (4); raízes e tubérculos (7); carnes e pescados (12); ovos, leite e queijos (9); hortaliças (21); frutas (26); açúcares e doces (10); óleos e gorduras (7); 
bebidas e diversos (9); enlatados e molhos (3); outros itens mencionados.

Na ocasião, foi perguntado sobre os alimentos adquiridos pela família nos 7 dias anteriores à entrevista, bem como a sua forma de aquisição, ou seja, compra, doações ou oriundos de lavoura ou criação. O entrevistador esperava a informação do entrevistado evitando a citação de alimentos, e, conseqüentemente, a indução da resposta. Antes de terminar a entrevista, o entrevistador observava se foram citados alimentos de todos os grupos. Na falta de algum alimento considerado de consumo habitual (Ex. açúcar, café, óleo e outros), o entrevistador poderia perguntar ao entrevistado se todos os alimentos adquiridos pela família nos últimos 7 dias foram citados durante a entrevista. Tendo em vista o número reduzido de alimentos disponíveis no domicílio, as informações foram coletadas em torno de 15 minutos.

Para o processamento dos dados, foi utilizado o programa Epilnfo, versão 6.04 (Epilnfo 6.04, WHO/CDC, Atlanta, GE, USA). A análise da disponibilidade dos alimentos nas famílias foi realizada para cada município separadamente, considerando como ponto de corte os alimentos citados em mais de $20 \%$ das famílias, analisados de acordo com a área geográfica. Os alimentos disponíveis em mais de $70 \%$ das famílias foram analisados de acordo com a escolaridade do chefe da família (anos de estudo) e o número de pessoas no domicílio.

Para verificar diferenças significantes da disponibilidade e tendências lineares de cada alimento nas categorias de área geográfica, escolaridade do chefe da família e número de pessoas no domicílio, utilizaram-se os testes de qui-quadrado e qui-quadrado para tendência, com níveis de significância de $5 \%(p \leq 0,05)$ e $1 \%$ $(p \leq 0,01)$.

Em atendimento ao disposto na Resolução 196/96 do Conselho Nacional de Saúde, esta pesquisa foi aprovada pelo Comitê de Ética do Instituto Materno Infantil Prof. Fernando Figueira, processo $n^{\circ} 386 / 2004$.
RESULTADOS

De acordo com os dados apresentados na Tabela 1, verifica-se um total de 33 alimentos disponíveis em $20 \%$ das famílias de Gameleira e de 30 alimentos, de São João do Tigre. Ao considerar como ponto de corte os alimentos que foram adquiridos por mais de $50 \%$ das famílias, esse número se restringe a apenas 16 e 15 alimentos em Gameleira e São João do Tigre, respectivamente.

Dentre os alimentos listados na tabela em referência, observa-se o açúcar como o alimento de maior disponibilidade nos domicílios, constando em mais de 93\% das famílias em cada um dos municípios, principalmente nas áreas rurais, apresentando resultado estatisticamente significante na área rural de Gameleira, em comparação à sua área urbana. Altos percentuais de famílias, em ambos os municípios, referiram o arroz e o feijão como alimentos existentes no domicílio, nos últimos sete dias anteriores à entrevista, sendo o arroz significantemente mais encontrado nas áreas rurais. Além deste, observa-se também, em Gameleira, uma alta disponibilidade de macarrão, principalmente na área rural $(p \leq 0,05)$. O feijão mostrou-se significantemente mais consumido na área rural de Gameleira. Outro alimento que chama a atenção é o óleo de soja, presente em elevados percentuais de domićlios nos dois municípios, com resultado significante para a área rural de São João do Tigre. Situação semelhante ocorreu em relação ao café, este com resultados significantes para as áreas rurais nos dois municípios.

Considerados por grupos, verifica-se um reduzido número de alimentos em Hortaliças e Frutas, nos municípios estudados, sendo alguns destes alimentos significantemente mais disponíveis na área rural de Gameleira. No grupo Cereais e Derivados, além do arroz, o fubá destaca-se como um dos alimentos mais disponíveis, principalmente no município da Zona da Mata (Gameleira, PE), tanto na área rural quanto na urbana. Entretanto, o pão foi citado mais freqüentemente pelas famílias do Semi-Árido (São João do Tigre, PB). Nos dois municípios, o pão foi significantemente mais disponível na área urbana do que na rural. 
Tabela 1. Disponibilidade relativa de alimentos em famílias dos municípios de Gameleira (PE) e São João do Tigre (PB), Nordeste do Brasil, 2005.

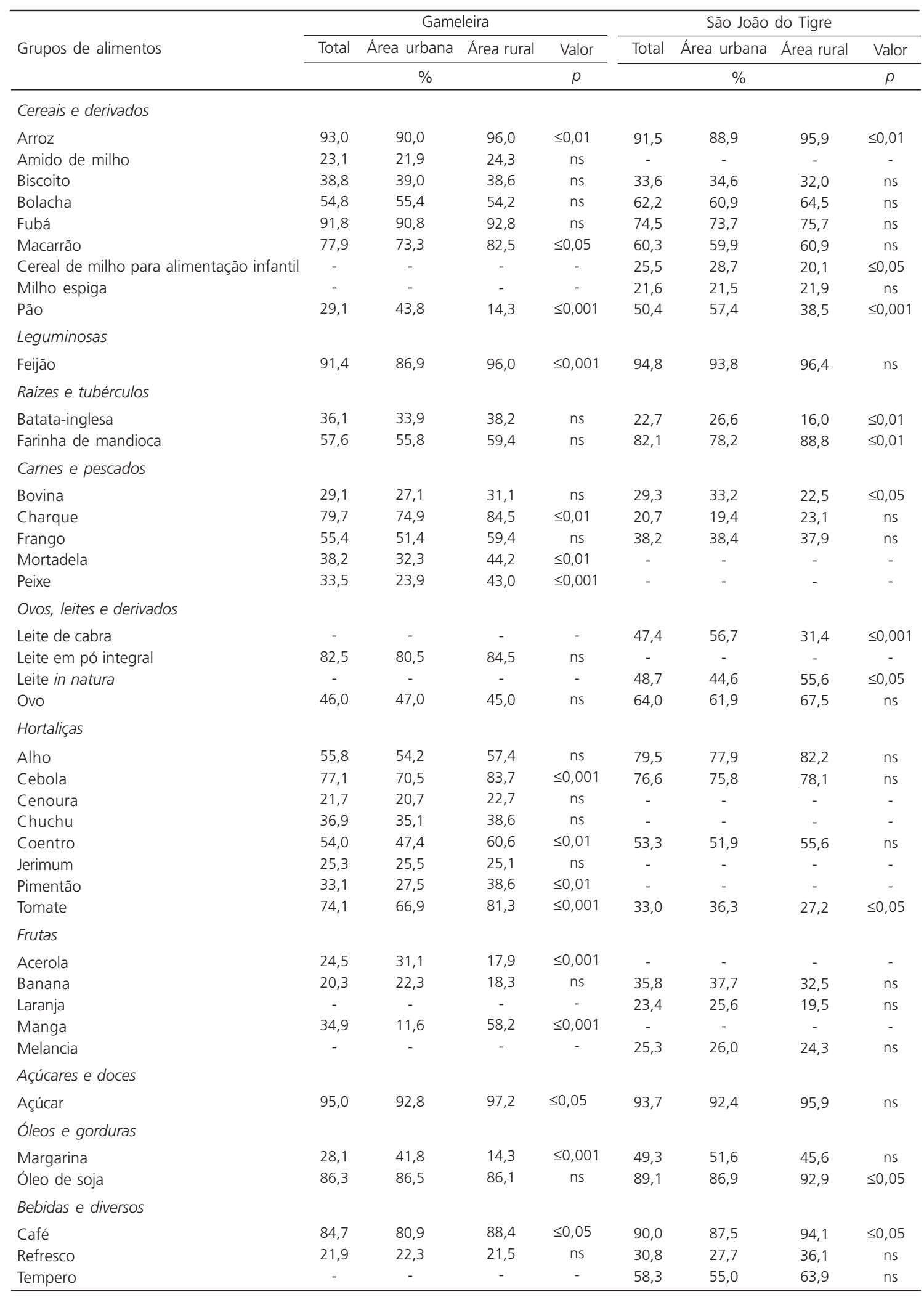

ns: não significante. 
A farinha de mandioca foi a maior representante do grupo Raízes e Tubérculos, principalmente no município do Semi-Árido, com significância estatística em favor da área rural.

No que diz respeito aos grupos de alimentos de origem animal (Carnes e Pescados, Ovos, Leites e Derivados), os resultados deste estudo indicam que na Zona da Mata, a charque e o leite de vaca encontravam-se disponíveis em percentuais acima de $70 \%$ dos domicílios. Diferenças ocorrem entre a Zona da Mata e o Semi-Árido, em que na primeira predominou o leite de vaca em pó integral enquanto que no Semi-Árido, o leite de vaca e de cabra in natura. No Semi-Árido se observam baixos registros dos alimentos destes grupos fontes de proteína animal, constando apenas o ovo como disponível em mais de $50 \%$ dos domicílios. Chama a atenção o leite de cabra, disponível em mais de $50 \%$ dos domićlios apenas da área urbana de São João do Tigre $(p \leq 0,001)$. Este foi significantemente mais disponível na área urbana, enquanto que o leite de vaca in natura, na área rural do município. No município de Gameleira, tanto a mortadela como o peixe mostraram-se significantemente mais disponíveis na área rural, sendo interessante notar a presença de mortadela em mais de 30\% dos domicílios.

Ao analisar a disponibilidade dos alimentos de acordo com a escolaridade do chefe da família (Figuras 1 e 2), categorizada em anos de estudo, observa-se que não houve diferença significante para a maioria dos alimentos disponíveis. Em Gameleira, houve diferença significante apenas para o café. Este alimento teve sua disponibilidade diminuída com o aumento do número de anos estudados pelo chefe da família. Em São João do Tigre, verifica-se diminuição esta-tisticamente significante de açúcar, feijão e café, à medida que aumentou a escolaridade do chefe da família.

Na associação entre a disponibilidade de alimentos e o número de pessoas no domicílio, diferenças significantes não foram encontradas para a maioria dos alimentos (Figuras 3 e 4). No município de Gameleira, somente o café demonstrou significância estatística positiva com o número de pessoas no domicílio, diferentemente de São João do Tigre, onde o açúcar, a farinha e o café apresentaram diferenças estatisticamente significantes.

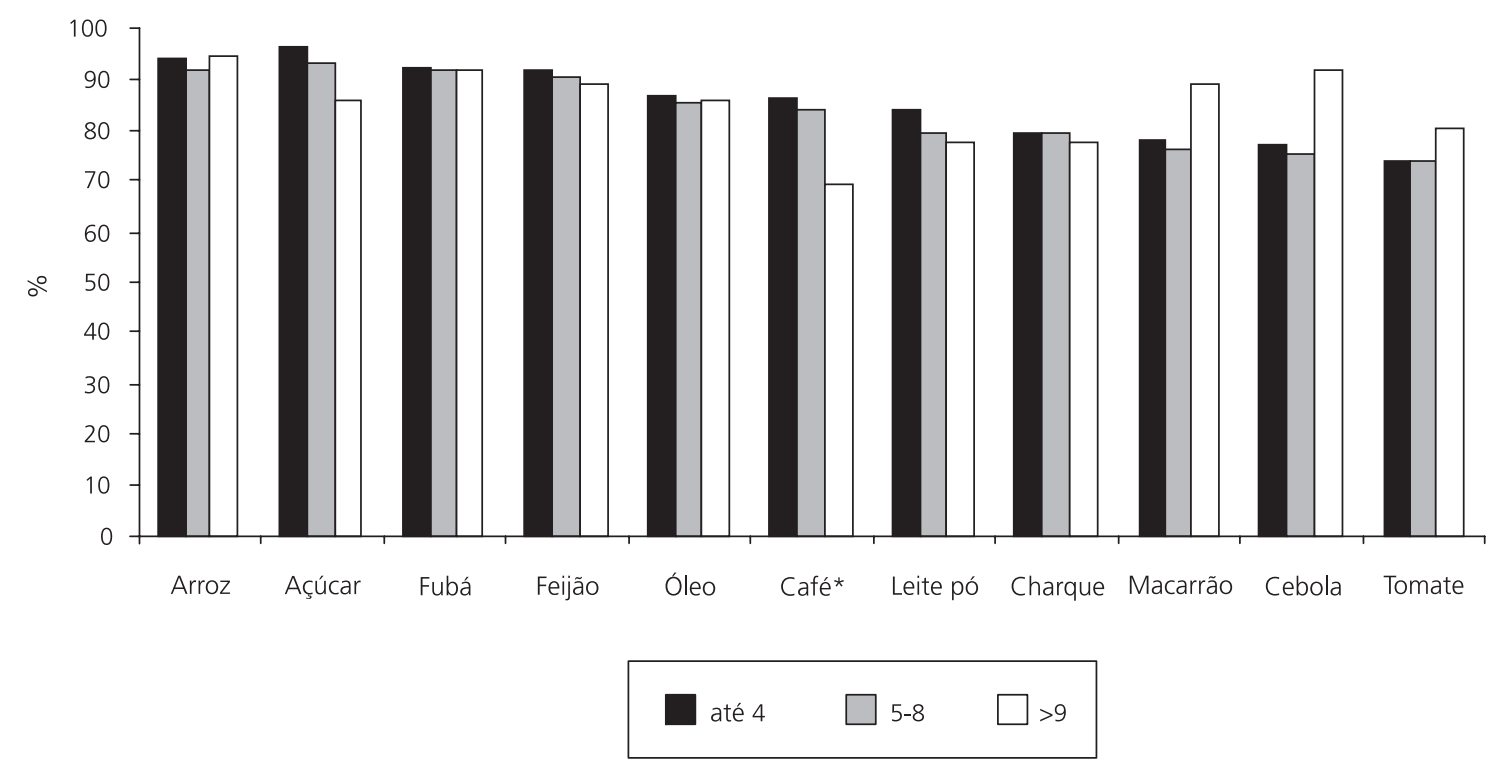

Figura 1. Distribuição percentual da disponibilidade de alimentos, segundo escolaridade do chefe da família (anos de estudo). Gameleira (PE), 2005.

Nota: ${ }^{*} p \leq 0,05$ 


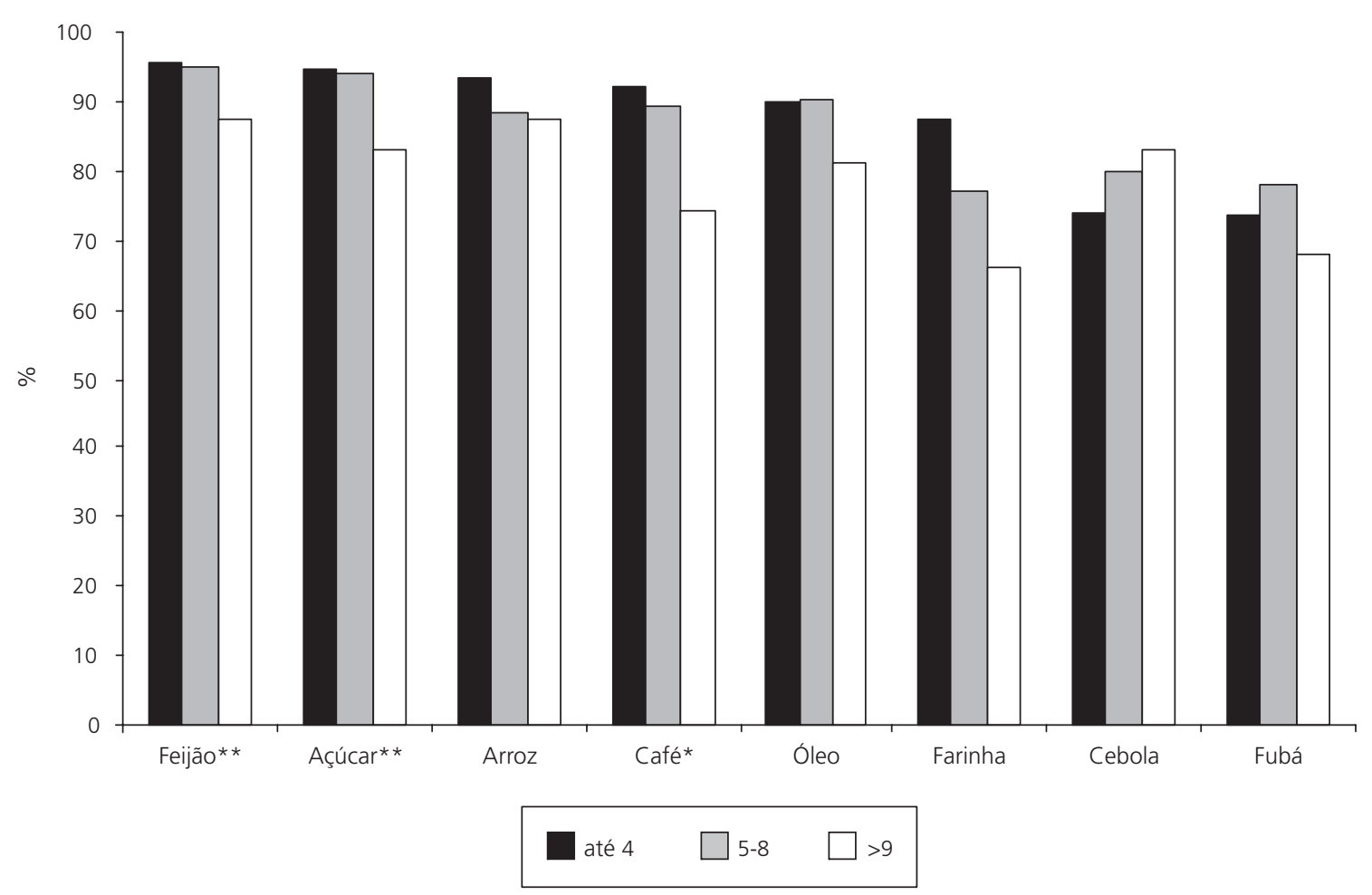

Figura 2. Distribuição percentual da disponibilidade de alimentos, segundo escolaridade do chefe da família (anos de estudo). São João do Tigre (PB), 2005.

Nota: ${ }^{*} p \leq 0,05 ;{ }^{*} p \leq 0,01$

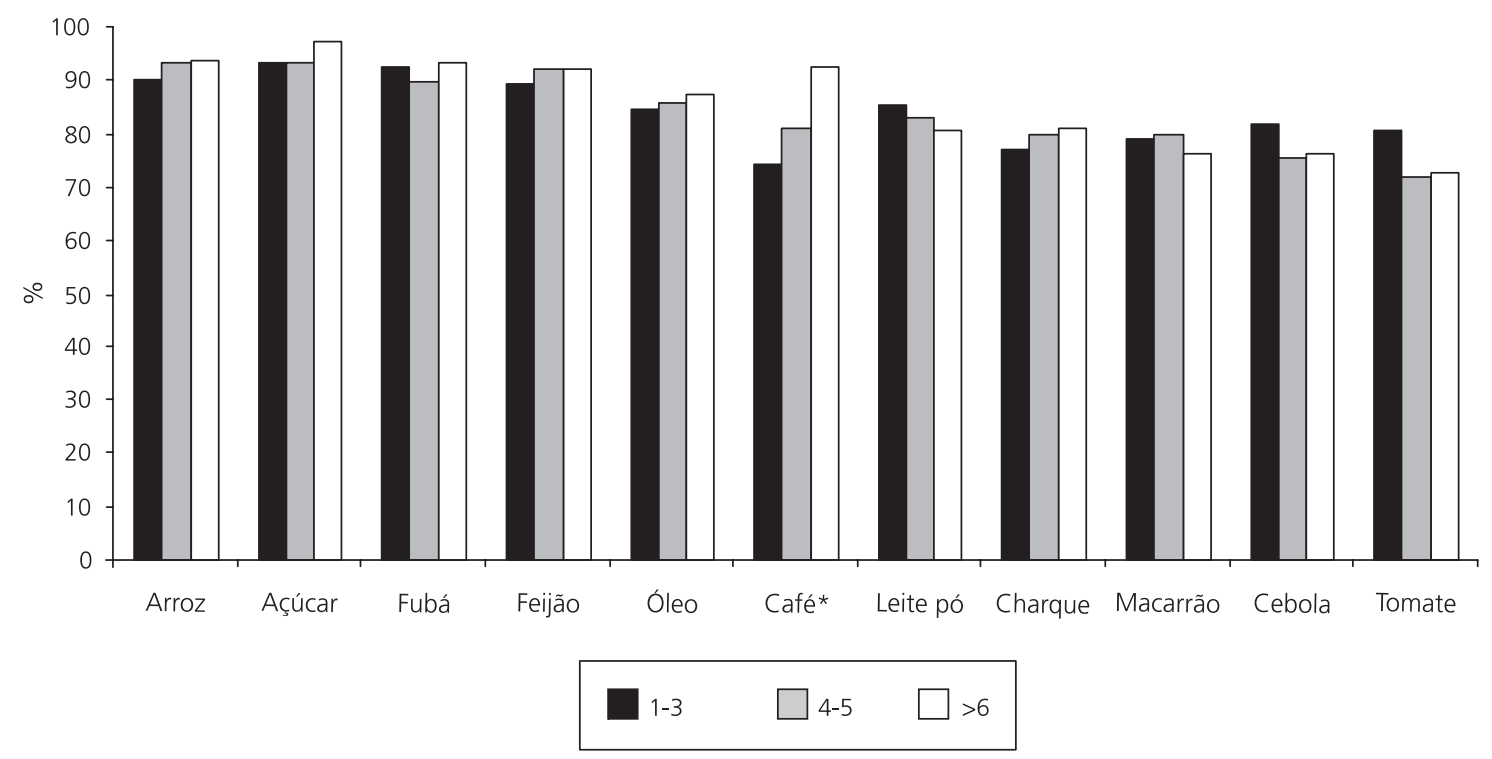

Figura 3. Distribuição percentual da disponibilidade de alimentos, segundo número de pessoas/domicílio. Gameleira (PE), 2005. Nota: ${ }^{*} p \leq 0,05$. 
326 | M.M. OSÓRIO et al.

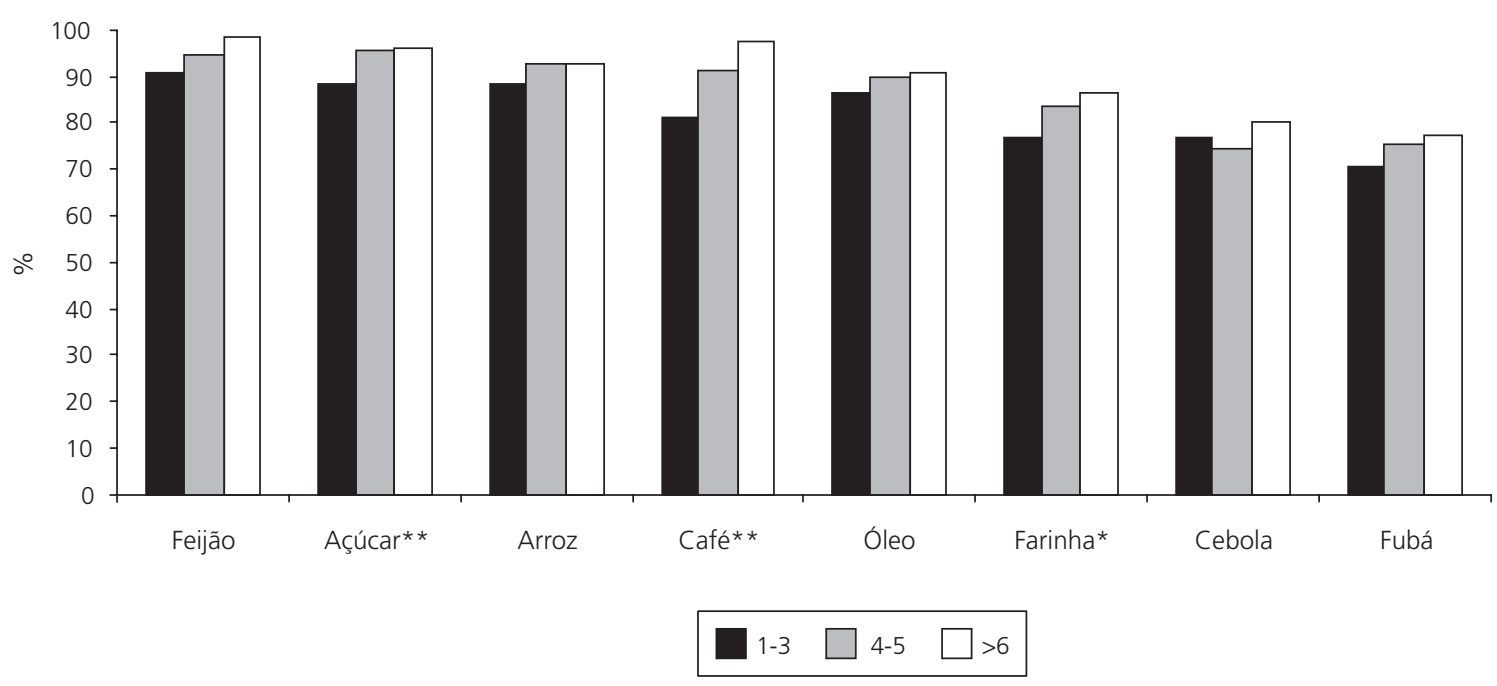

Figura 4. Distribuição percentual da disponibilidade de alimentos, segundo número de pessoas/domicílio. São João do Tigre (PB), 2005.

Nota: ${ }^{*} p \leq 0,05 ; * * p \leq 0,01$.

DIS CUSS Ã O

O destino das nações é, em parte, determinado pelo padrão de alimentação da população e a análise alimentar denuncia as diferentes faces de uma má nutrição em que, alcançados os vários estágios fisiológicos, pode culminar com a exclusão social ou mesmo a morte dos indivíduos afetados $^{9,10}$.

No Brasil, apesar do crescente aumento na produção de alimentos, $27 \%$ da população, nas áreas urbanas, não têm renda suficiente para garantir uma alimentação satisfatória, enquanto nas áreas rurais, este valor sobe para $47 \%{ }^{3}$.

De acordo com os resultados deste trabalho (Tabela 1), verifica-se que 24 alimentos são de uso comum nas duas regiões do estudo, 12 dos quais (arroz, fubá, macarrão, pão, feijão, batata inglesa, farinha de mandioca, frango, ovo, leite, açúcar, óleo vegetal) também figuram na relação daqueles obtidos em inquéritos nacionais realizados pelo Instituto Brasileiro de Geografia e Estatística, entre o período de 1974 a $2003^{3}$ e no Estudo Multicêntrico ${ }^{11}$, sobre consumo alimentar em cinco cidades brasileiras.
Embora se constate limitada variação de alimentos em ambas as regiões estudadas, verifica-se um aumento no número de alimentos disponíveis por domicílio, se comparados com os resultados da II Pesquisa Estadual de Saúde e Nutrição ${ }^{12}$, a qual demonstrou que apenas 10 alimentos eram consumidos por mais de $50 \%$ da população.

A primazia do arroz e do feijão na dieta do brasileiro, cuja combinação é tida como prato típico nacional, e base da alimentação no Brasil, é ratificada neste estudo, tendo em vista o elevado percentual de famílias que os têm disponíveis, posto que os menores valores foram $88,9 \%$ para o arroz e $86,9 \%$ para o feijão. Tais dados mostram tendência contrária ao observado nas regiões economicamente mais desenvolvidas do País, em que se verifica um declínio no consumo destes alimentos ao longo das últimas décadas ${ }^{13}$. Apesar disso, o macarrão tem presença marcante nos domicílios do município de Gameleira (77,9\%), se comparado com o município de São João do Tigre $(60,3 \%)$.

O fato de o fubá estar mais presente nos domicílios da Zona da Mata permite inferir o seu 
uso como substituto do pão. Interessante notar ainda, no grupo de Raízes e Tubérculos, a baixa freqüência da batata-inglesa e a ausência de macaxeira, inhame e batata-doce, alimentos tradicionais no Nordeste do País, o que confirma mudanças no padrão alimentar das populações, inclusive daquelas de baixo poder aquisitivo².

No que se refere aos grupos de alimentos de origem animal, destaca-se considerável disponibilidade de charque na região da Zona da Mata em relação ao Semi-Árido e ainda o consumo de mortadela, presente apenas na primeira região, alimentos com altas concentrações de sal. Salienta-se ainda que o leite de cabra, em São João do Tigre, é produto da pecuária, beneficiado pela cooperativa local e distribuído como um programa de governo do município.

Nos grupos de Frutas e Hortaliças, consideradas veículo de vitaminas e minerais, as maiores freqüências de disponibilidade são para alho, cebola, coentro e tomate, todavia utilizados em pequeníssimas quantidades, com a finalidade de tempero.

No Brasil, dados de consumo estimados pelas pesquisas nacionais nas últimas três décadas, já mostravam declínio importante no consumo de frutas e hortaliças em áreas metropolitanas brasileiras ${ }^{2}$. Na Pesquisa de Orçamentos Familiares de 2003 identifica-se a diminuição no consumo de frutas e hortaliças em todas as regiões do Brasil, tanto na área urbana, quanto na rural, constatando serem alimentos historicamente consumidos em quantidades inadequadas no Brasil ${ }^{3}$.

Os resultados do presente estudo corroboram as pesquisas de consumo alimentar realizadas nacionalmente, as quais concluem que populações de regiões com baixos índices de desenvolvimento apresentam consumo relativamente menor de proteína de origem animal e têm como base da alimentação os grupos de cereais e leguminosas, em detrimento de frutas e hortaliças. Nas duas localidades estudadas, os efeitos estruturais da pobreza se acentuam nos períodos da entressafra da cana-de-açúcar e de estiagem, respectivamente na Zona da Mata e no Semi-
-Árido Nordestino, agravando as condições de insegurança alimentar e nutricional. Todavia a disponibilidade de alimentos energéticos como açúcar e gordura e ainda de frutas, legumes e verduras, parece acompanhar o que acontece nas regiões mais desenvolvidas do País, evidenciando a generalização da transição nutricional, independentemente da área geográfica e da condição socioeconômica ${ }^{11,13}$.

Frente aos baixos Índices de Desenvolvimento Humano apresentados pelos dois municípios pesquisados, os anos de estudo do chefe da família e o número de pessoas no domicílio foram utilizados como indicadores socioeconômicos para verificação de diferenças na disponibilidade de alimentos entre os domicílios estudados. Conforme dados apresentados nas Figuras 1, 2, 3 e 4, verifica-se que apenas alguns alimentos mostraram diferenças significantes em relação a estes dois indicadores selecionados.

Nota-se, no município de Gameleira, um aumento da disponibilidade de hortaliças (cebola e tomate) nas famílias cujo chefe possuíam maior nível de escolaridade. Sabe-se que a escolaridade aumenta proporcionalmente com a renda dos membros das famílias e esta última tem sido associada ao aumento da participação de certos grupos alimentares na dieta, principalmente os grupos fontes de proteína animal, frutas e hortaliças, mostrando que a renda pode interferir na seleção qualitativa de alimentos, o que, conseqüentemente, aumenta o consumo de micronutrientes essenciais ${ }^{13-15}$.

No município de São João do Tigre, parece haver uma racionalidade da dieta em termos de disponibilidade de alimentos mais energéticos e, conseqüentemente, de menor preço, em função de piores condições socioeconômicas, tendo em vista ser observada uma relação direta entre a disponibilidade de farinha e açúcar e o número de membros no domicílio, e uma relação inversa entre a disponibilidade de açúcar e feijão e a escolaridade do chefe da família.

O café, disponível em elevado número de domicílios nos dois municípios, foi um alimento 
que mostrou redução significante com o aumento do nível de escolaridade do chefe da família, em ambas as regiões estudadas, sugerindo a substituição deste por outro tipo de bebida. Todavia foi identificado em São João do Tigre um aumento da disponibilidade do café, em função do maior número de pessoas no domicílio.

A análise dos dados de disponibilidade de alimentos do presente estudo pode ter sofrido limitação por conta da coleta dos dados, que requereu um esforço de memória dos últimos 7 dias anteriores à entrevista. Na tentativa de reduzir o viés de memória, os entrevistadores faziam a revisão do formulário do inquérito, logo após o preencher com as informações fornecidas pelo entrevistado, com a finalidade de averiguar a falta de alimentos conhecidamente pertencente ao consumo habitual da população. Além disso, por se tratar de populações com baixo poder de compra, a aquisição de alimentos estava condicionada a períodos semanais e com pouca variedade, o que se espera tenha contribuído na redução do viés de memória.

Diante do exposto, os dados apresentados, oriundos de municípios da Região Nordeste, com baixos Índices de Desenvolvimento Humano, mostram que a dieta da população das duas áreas geográficas estudadas caracteriza-se por um número reduzido de alimentos, com maior disponibilidade de alimentos de origem vegetal e de alto valor energético, além de escassa presença de hortaliças e frutas. A disponibilidade domiciliar de alimentos sofreu poucas variações, em decorrência das condições do número de pessoas no domicílio e da escolaridade do chefe da família.

O perfil da disponibilidade de alimentos nestes municípios proporciona uma visão realista de insegurança alimentar a que estão submetidas estas populações, o que pode explicar o aparecimento de distúrbios nutricionais e, conseqüentemente, de doenças crônicas não transmissíveis $^{4,16}$. Apesar da limitação econômica, os recursos disponíveis poderiam ser melhor aproveitados nestas regiões, tendo em vista que alimentos regionais, como macaxeira, batata-doce, miúdos, não se encontravam presentes nos domicílios. Dessa maneira, o conhecimento da disponibilidade de alimentos se faz necessário para a implantação de políticas específicas voltadas para a produção e abastecimento de alimentos, emprego e renda, saúde, nutrição e alimentação.

A educação nutricional, como uma estratégia efetiva, torna-se imprescindível na Política Nacional de Segurança Alimentar, com o objetivo coletivo de ampliar a questão alimentar, nutricional e de saúde no contexto socioeconômico e demográfico da região, contribuindo para o desenvolvimento sustentável destas populações ${ }^{17}$.

Neste sentido, o estímulo ao aumento da disponibilidade de alimentos, em termos qualitativo e quantitativo, com vistas ao consumo equilibrado de maior variedade destes, deve ser atualmente enfatizado nos esforços governamentais empreendidos no combate à fome. Para tanto, urge uma maior articulação de múltiplos setores, em âmbito nacional, incluindo governo, indústria, mídia, profissionais de saúde e sociedade civil organizada, como instrumento fundamental para a captação e a ampliação de recursos financeiros, além de capacitação de recursos humanos, visando à implantação de programas e estratégias de sucesso no combate aos problemas alimentares e nutricionais no Brasil.

\section{A GRADECIMENTO}

Ao Conselho Nacional de Desenvolvimento Científico e Tecnológico, pelo apoio financeiro para a realização desta pesquisa (Processos nos 502952/03-2 e 502955/03-1).

\section{O LABORADORES}

M.M. OSÓRIO e M.A. RIBEIRO participaram da elaboração dos projetos de estudo e dos instrumentos de coleta de dados; da supervisão dos trabalhos de campo; do levantamento bibliográfico; da análise estatística e da redação cientifica do artigo. E.C. COSTA e S.P.O. SILVA participaram da elaboração dos instrumentos de coleta de dados; da coleta e tabulação 
dos dados de campo; do levantamento bibliográfico, da análise estatística e da redação científica do artigo. C.F. ESTEVAM participou da tabulação dos dados de campo e da redação científica do artigo.

\section{REFERÊ NCIAS}

1. Mondini L, Monteiro CA. Mudanças no padrão de alimentação da população urbana brasileira (1962-1988). Rev Saúde Pública. 1994; 28(6): 433-9.

2. Monteiro CA, Mondini L, Costa RBL. Mudanças na composição e adequação nutricional da dieta familiar nas áreas metropolitanas do Brasil (1988-1996). Rev Saúde Pública. 2000; 34(3): 251-8.

3. Instituto Brasileiro de Geografia e Estatística. Pesquisa de orçamentos familiares 2002-2003. Análise da disponibilidade domiciliar de alimentos e do estado nutricional no Brasil. Rio de Janeiro: IBGE; 2004.

4. Organización Mundial de la Salud. Informe sobre la salud en el mundo 2002: reducir os riesgos y promover uma vida sana. Ginebra: OMS; 2002.

5. World Heath Organization. Global strategy on diet, physical activity and health. Fifty seventh world health assembly [WHA 57.17]. Geneva: WHO; 2004.

6. Brasil. Ministério da Saúde. Análise da estratégia global para alimentação saudável, atividade física e saúde. Portaria do Ministério da Saúde n. 596, de 8 de abril de 2004. Brasília, 2004 [acesso 2006 jun 10]. Disponível em: <http://www.saude.gov.br/ nutricao/publicacoes $>$.

7. Batista Filho M. Sustentabilidade alimentar do semi-árido brasileiro. Recife: IMIP; 2005. Série Publicações Científicas Instituto Materno Infantil Prof. Fernando Figueira - IMIP, n.11.

8. Programa das Nações Unidas para o Desenvolvimento. Atlas de desenvolvimento humano [acesso
2000 abr 15]. Disponível em: <http://www.pnud. org.br>.

9. Weisburguer JH. Eat to live, not live to eat. Nutrition. 2000; 16(9):767-73.

10. Food and Agriculture Organization. El estado de la inseguridad alimentaria en el mundo 2006: la erradicación del hambre en el mundo evaluación de la situación diez años después de la Cumbre Mundial sobre la Alimentación. Rome: FAO; 2006.

11. Galeazzi MAM, Domene SMA, Sichieri R. Estudo multicêntrico sobre consumo alimentar. Campinas: Unicamp; 1997. Caderno Especial do NEPA.

12. Batista Filho M, Romani SAM. Alimentação, nutrição e saúde no estado de Pernambuco: espacialização e fatores socioeconômicos. Recife: IMIP; 2002. Série Publicações Científicas do Instituto Materno Infantil de Pernambuco, IMIP. n.7.

13. Levy-Costa RB, Sichieri R, Pontes NS, Monteiro CA. Disponibilidade domiciliar de alimentos no Brasil: distribuição e evolução (1974-2003). Rev Saúde Pública. 2005; 39(4):530-40.

14. Lima ES, Euclydes MP, Cruz TA, Casali AD. Condições sócio-econômicas, alimentação e nutrição da população urbana de uma localidade do estado de Minas Gerais (Brasil). Rev Saúde Pública. 1989; 23(5):410-21.

15. Enes CC, Silva MV. Disponibilidade de energia e nutrientes nos domicílios brasileiros no início do século XXI. J Braz Soc Food Nutr. 2006; 31(1): 17-32.

16. Monteiro CA, Benício MHDA, Conde WL, Popkin BM. Shifting obesity trends in Brazil. Eur J Clin Nutri. 2000; 54(4):342-6.

17. Brasil. Ministério da Saúde. Guia alimentar para a população brasileira: promovendo a alimentação saudável. Brasília: Ministério da Saúde; 2006. Série A. Normas e Manuais Técnicos.

Recebido em: 15/8/2007

Versão final reapresentada em: 24/10/2008

Aprovado em: 30/3/2009 